\title{
Space mission resilience with inter-satellite networking
}

\author{
Christopher J. Lowe* and Malcolm Macdonald. ${ }^{\dagger}$ \\ Department of Mechanical and Aerospace Engineering, James Weir Building, 75 Montrose Street, \\ University of Strathclyde, Glasgow, G1 1XJ
}

Keywords: Satellite; Space; Networking; Markov; Monte-Carlo; Value; Failure; Anomaly

Declarations of interest: none

\begin{abstract}
Satellites typically operate in isolation from their orbiting counterparts, but communicating only with ground-based infrastructure leaves them susceptible to the consequences of on-board anomalies. Loss of payload, communication system, or other sub-system function could render the entire satellite inoperable. This susceptibility can be partially mitigated through the addition of an inter-satellite networking capability, which offers value in terms of increased general performance and an increased resilience to on-board anomalies. While a typical platform can be modelled to exhibit only two fundamental states: operational and failed, a networking-capable platform (specifically one with an inter-satellite communication capability) exhibits six states, each reached through a unique combination of sub-system malfunctions. The result of this added resilience is a reduction in the likelihood of the satellite reaching a fully-failed state. Simulations for independent and networking-capable systems are presented that illustrate the benefits and limitations of intersatellite networking in terms of failure resilience. It is shown that whilst a networked system can be expected to reach greater levels of performance utility, sub-system anomalies are found to result in greater percentage levels of performance degradation compared to a non-networking-capable system with similar characteristics.
\end{abstract}

\footnotetext{
${ }^{*}$ Research Fellow, christopher.lowe@strath.ac.uk (corresponding author)

† Professor of Space Technology, malcolm.macdonald.102@strath.ac.uk
} 


\section{Introduction}

Failures can, and do, occur on space systems, sometimes with serious consequences. Be it at a component, sub-system or platform*-level, anomalies in space are often difficult to resolve, especially if they occur in hardware. The impact of inter-satellite networking (ISN) capability, and how it can affect a platform's resilience in the event of system anomalies and degraded performance is investigated. A model is developed to enable analysis of the effect of loss of function of various on-board systems, giving an understanding of platform state transitions over time.

ISN can be defined as the transfer of information between spacecraft via inter-satellite links (ISLs). While the concept of ISN is not new, the investigation of its effect on a platform's resilience (specifically, its ability to mitigate performance degradation as a result of on-board anomalies) has not received much attention, and as such the actual value of ISN is poorly understood. In [1], platform anomalies are considered in the data routing process, such that the ISN path-planning is updated based on changes to the network, however a simplified approach to failure probability (exponential) is taken and an assessment of the impact on overall mission value is investigated. Other works evaluate either ISN [2]-[4], or reliability analysis in spacecraft [5]-[8], in some detail, but do not offer insights into the link between these phenomena. This work aims to overcome this omission and consider a realistic anomaly probability and the effectiveness of ISN to overcome the associated loss in value.

Discrete-time Markov chains are used to describe the transition between states, as a consequence of loss of function, where a platform with an ISN capability exhibits degraded (partially-failed) states not apparent on non-ISN capable platforms. These additional states provide graceful degradation of the individual platform by increasing the likelihood that a platform will exhibit some useful functionality for longer periods of its lifetime. Following this, the problem is investigated at the network-level, whereby utility of a space network subject to platform sub-system failure over its lifetime is analysed. Using the Markov chain model and time-dependent failure probability a semi-analytical approach is introduced in this work to illustrate lifetime utility degradation, and is exercised through Monte Carlo simulation methods.

A point design, that is, one that is rigid in its characteristics and operation, may achieve optimal performance in the specific conditions for which it was developed, but may be left exposed in the event of anomalies. Consideration of uncertainties as part of the design process, in particular from the point of view of failure (reliability engineering), is not new and has been practiced since the early 1900s [9]. However, studies into the effects resulting from arbitrary uncertain events taking place have only recently come to the fore [10]-[12]. It is these effects from uncertainty that impact a system's operational capabilities, which can be often translated into a change in utility.

Uncertainty can be categorised into two forms; i) Aleatoric - that which cannot be known a priori, such as minor fluctuations in atmospheric conditions or the time of future solar activity, and ii)

\footnotetext{
* The term "platform" shall be used to refer to an individual spacecraft or satellite throughout this article.
} 
Epistemic - that which occurs due to lack of knowledge, such as the centre of mass of an instrument or the cost of a launch. Here, while both are acknowledged as important and valid, only the former (aleatoric) will be considered. Irrespective of the type of aleatoric uncertainty (e.g. failure, market change, environmental change, technology obsolescence), the over-arching effect is generally a reduction in system utility, or performance. Measurement of this utility change allows us to better understand a system's lifetime behaviour and the value it can offer over this period.

\subsection{Markov Chains}

Markov chains offer a convenient way of modelling the stochastic process of component/system failure and allow evaluation of expected state evolution over time. A Markov process is a stochastic process defined by a set of random variables $\{X(t), t \in T\}$, where each $X(t)$ is a "state", defined on some probability space, at time $t$. $T$ is the time horizon, where $T=\{0,1,2, \ldots\}$ forms a discrete time Markov chain (DTMC), and $T=\{t: 0 \leq t<\infty\}$ forms a continuous time Markov chain (CTMC). For the purposes of this work, the focus shall be on DTMCs due their applicability to systems for which the probability of transitioning between states may be time-varying [13], which is the case for space systems.

The Markov property is analogous to the memoryless property, such that transition from one state to another depends only on the current state, and not on states in which the system resided during some previous time. Formally, this is

$$
\operatorname{Prob}\left\{X_{n+1}=x_{n+1} \mid X_{n}=x_{n}, X_{n-1}=x_{n-1}, \ldots, X_{0}=x_{0}\right\}=\operatorname{Prob}\left\{X_{n+1}=x_{n+1} \mid X_{n}=x_{n}\right\},
$$

where $x_{i}$ is the state of the system at time $i$. The above can be simplified to

$$
p_{i j}(n)=\operatorname{Prob}\left\{X_{n+1}=j \mid X_{n}=i\right\}
$$

Given a discrete and finite set of states, the matrix $P(n)$, in which $p_{i j}(n)$ is the entry in the $i^{\text {th }}$ row and $j^{\text {th }}$ column, is called the transition probability matrix, and contains the probability of transitioning between any two states at time $n . P(n)$ is written as

$$
P(n)=\left[\begin{array}{cccc}
p_{11}(n) & p_{12}(n) & \cdots & p_{1 K}(n) \\
p_{21}(n) & p_{22}(n) & \cdots & p_{2 K}(n) \\
\vdots & \vdots & \ddots & \vdots \\
p_{K 1}(n) & p_{K 2}(n) & \cdots & p_{K K}(n)
\end{array}\right]
$$

where $K$ is the number of discrete states and thus $P(n)=\mathbb{R}^{K \times K}$. It follows that

$$
\sum_{\text {all } j} p_{i j}(n)=1, \forall i
$$


where $i=j$ is a possible scenario and simply represents the absence of any transition from the current state. In a (time-)nonhomogeneous DTMC, $P(n)$ may vary with $n$, which is an important characteristic to capture for systems that suffer from either reliability decay over time, or a higher failure probability at the beginning of life. Analysis into the robustness/survivability achieved from swarm size [5] and system fractionation [14] has been conducted using a homogeneous DTMC, which results in this time-varying phenomena being missed. In [15] and [8] it is shown that this time-varying property is real for space systems and should be incorporated.

For systems that do not undergo servicing or repair, applicable to the vast-majority of satellites, transition from a failed state back to an operational one is not possible, such that the $1{ }^{\text {st }}$ column in $P(n)$ is $\left[p_{11}(n) \quad 0 \quad 0 \quad 0^{T}\right.$ and the final row (fully failed) is $\left[\begin{array}{llll}0 & 0 & 0 & 1\end{array}\right]$, which represents an absorbing state. As an example, Figure 1 illustrates a typical Markov chain with four states, in which state 2 always transitions (no arc to itself) and state 4 is absorbing.

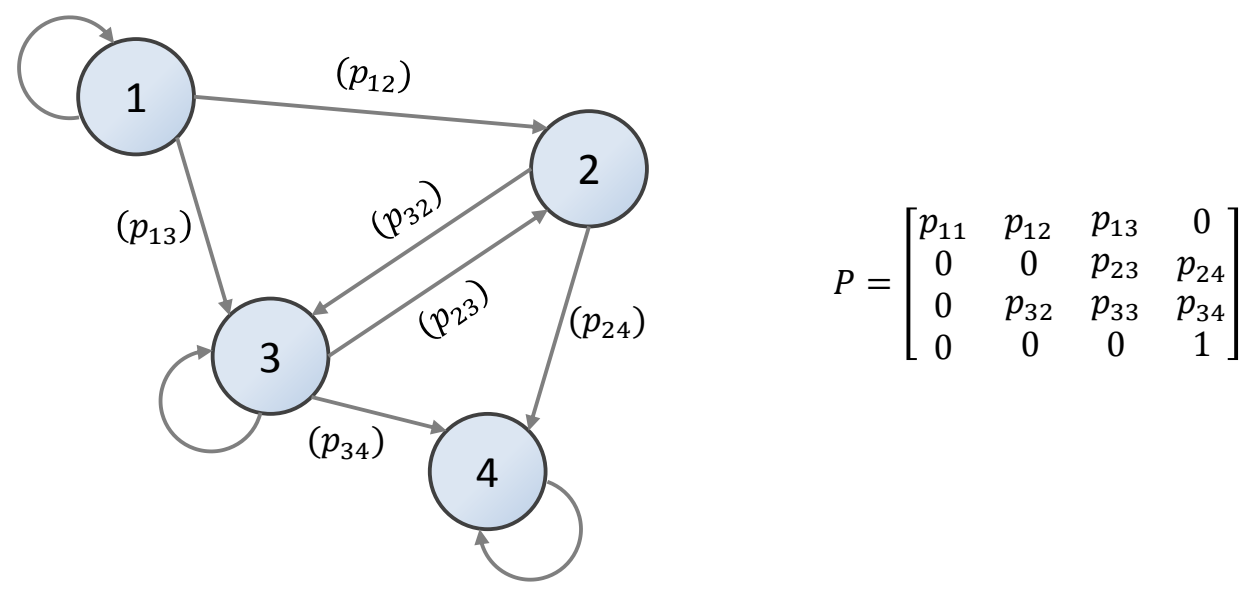

Figure 1 - Example of a DTMC with an absorbing state

In order to identify the expected utility being offered by a system subject to stochastic failures over its lifetime, transient analysis should be conducted, which provides an answer to the question "what is the probability that our system will be in state $x$ afterm time steps, given that it is in state $y$ now'? The Chapman Kolmogorov equations offer such functionality, derived from the fact that the probability for a two-step transition from state $i$ to $k$ is

$$
\begin{gathered}
\operatorname{Prob}\left\{X_{n+2}=k \mid X_{n+1}=j, X_{n}=i\right\}=p_{i j} p_{j k}, \forall j \in\{1,2, \ldots, K\} \\
\operatorname{Prob}\left\{X_{n+2}=k \mid X_{n}=i\right\}=\sum_{\text {all } j} p_{i j} p_{j k}
\end{gathered}
$$

which is the $i k^{\text {th }}$ element in $P^{2}$ for a homogenous DTMC, or the $i k^{\text {th }}$ element in $P^{(n+2)}(n, n+1, n+$ $2)=P(n+2) P(n+1) P(n)$ in a non-homogeneous DTMC. This can be generalised to obtain the Chapman Kolmogorov equation for non-homogeneous DTMCs, as 


$$
P^{(m)}(n, n+1, \ldots, n+m-1)=P(n) P(n+1) \ldots P(n+m-1)
$$

Finally, given a row vector $\pi^{(n)}=\mathbb{R}^{1 \times K}$ describing the probability of being in state $i \in\{1,2, \ldots, K\}$ at some time $n$, the probability of being in state $i$ at time $(m+n)$ is

$$
\pi^{(m+n)}=\pi^{(n)} P(n) P(n+1) \ldots P(n+m-1),
$$

which is fundamental in understanding the answer to the above question of future state, based on current state.

\subsection{Anomalies on Space Systems}

Complete failure affects approximately $6 \%$ of satellites within their first 7 years and almost $9 \%$ of satellites within their first 12 years (Figure 2, non-parametric data) [15]. Furthermore, in recent years there has been an increase in the use of satellites adhering to the CubeSat standard [16], a bus-type that tends to exhibit lower levels of reliability compared to the larger counterparts, as evidenced in the CubeSat Failure Database in [17]. Failure is therefore not something that can be ignored and both the likelihood of its arrival and its effect on mission value should be understood and indeed accounted for in the design process. Exactly how a particular sub-system fails can be considered arbitrary for the purposes of this work, however it is important to understand the likelihood of failure occurring over a particular timeframe. It has been shown in [7], [15], [17] and [18] that satellite sub-system failure can be approximated to a Weibull distribution, capturing the higher probability of failure at beginning of life that is neglected when using other functions, such as a linear regression. 
The reliability of a traditional space platform, or in other words its probability of residing in a nonfailed state, is shown in Figure 2.

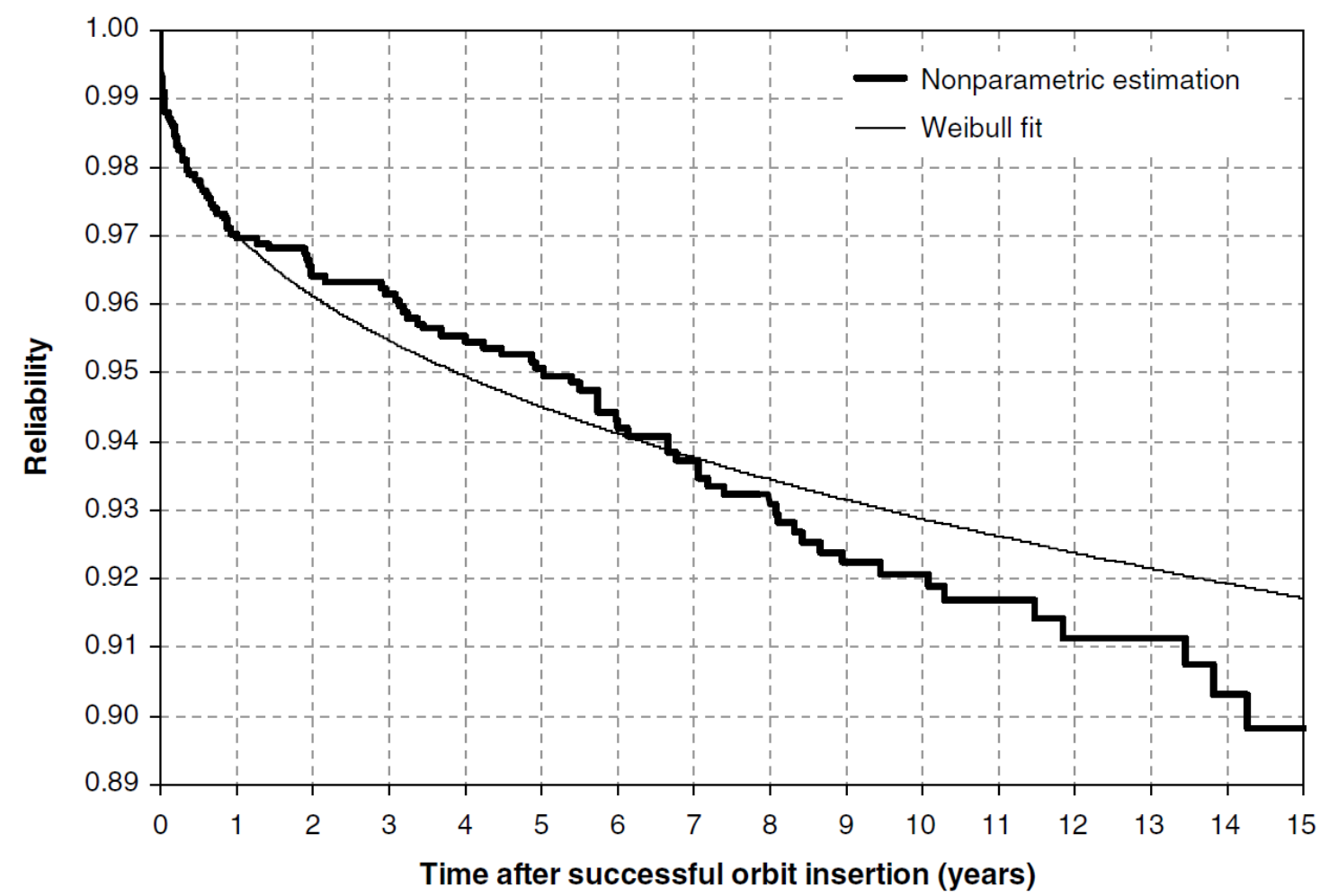

Figure 2 - Weibull distribution correlation to estimated reliability based on actual satellite data[15]

The operation of a traditional, monolithic* satellite can typically be reduced to that of data collection via its payload/s, and data delivery via its communication system/s. Failure of either of these sub-systems, or a supporting element (e.g. power system or on-board computer), would result in its general functionality not being realised and thus it would transition into a failed state. In reality, there exist various intermediate, partially-failed states following partial-failure of a subsystem due to failure or degradation at the component level ${ }^{\dagger}$, but for the purposes of this work, and to more clearly illustrate the effect of a networking capability, these are omitted.

\subsection{Utility}

Utility can be defined as the measure of effectiveness held by a single attribute, or set of attributes, such that a high utility is preferred from the point of view of performance. While attributes are described in terms of their associated units and quantity, utility offers a normalised quantification

\footnotetext{
${ }^{*}$ A monolithic satellite is designed to operate in isolation from other satellites, i.e. it does not rely on one or more other spacecraft for a critical function (e.g. data download)

† Consider, for example, failure of a string of solar cells or degraded attitude controller. The result would likely be a reduction in payload duty-cycle, such that some utility can still be maintained, but at a lower level than if fully operational
} 
between attributes that can be used for direct comparison and/or combination. For example, in the case of Earth observation, spatial resolution of an image is measured in meters and is generally minimised for best performance, while revisit rate (i.e. the number of views over a target, per unit time) is measured in frequency, and is generally maximised. By mapping these attributes to a normalised utility, which has a pre-defined maximum and minimum bound, they can be compared directly, with each having a value of between 0 (worst) and 1 (best). Furthermore, by scaling and combining multiple attributes, using methods from multi-attribute utility theory (MAUT) [19], it is possible to exploit a single utility metric that describes the overall performance of the system. This approach has been used to good effect in the aerospace community as a way to combine attributes and better understand stake-holder preferences during trade-space exploration exercises [20].

There exist a number of underlying axioms and assumptions that must be understood before implementing MAUT. These include attribute transitivity, e.g. if $X>Y$ and $Y>Z$, then $X>Z^{*}$, and the level of independence (preferential, utility and additive) with which attributes can be treated with respect to each other.

On the independence parameters, Preferential independence is where an attribute's preference is unchanged when considered alongside any other attribute. If $X$ and $Y$ are two attribute sets, then if $\left(X_{1}, Y_{1}\right)>\left(X_{2}, Y_{1}\right)$, preferential independence would dictate that $\left(X_{1}, Y_{2}\right)>\left(X_{2}, Y_{2}\right)$ always. Utility independence is where the utility of an attribute in $X$ remains unchanged for varying attributes in $Y$. And, additive independence is a characteristic whereby the decision maker expresses indifference to combinations of different attributes of a particular utility. If $X$ and $Y$ are two attribute sets and if $\left(X_{1}, Y_{1}\right)=\left(X_{2}, Y_{2}\right)$, then additive independence would dictate that $\left(X_{1}, Y_{2}\right)=$ $\left(X_{2}, Y_{1}\right)$. In other words, if we are indifferent to choosing $X_{1}$ over $X_{2}$, when combined with $Y_{1}$ and $Y_{2}$ respectively, then we remain indifferent should they be paired with $Y_{2}$ and $Y_{1}$ respectively.

While a general form for multi-attribute utility (MAU) calculation does exist, the multiplicative form offers a simple and often acceptable approach, for which preferential and utility independence must be demonstrated. Moreover, the presence of additive independence allows use of the simpler, additive form. These independence criteria, along with decision-maker preferences, are typically derived through interviews, where lottery questions ${ }^{\dagger}$ are posed to identify the stakeholder preferences with respect to individual, and combinations of, attributes. From this information, it is possible to derive single-attribute utility (SAU) functions, which represent the utility $(u)$ as a function of each attribute $x$, where $u(x) \in[0,1], \forall x$.

\footnotetext{
* the symbol $>$ denotes preference of the term on the left hand side over that on the right hand side

+ Consider the case where one can choose between having either a 50\% chance of winning $\$ 100$ (and a 50\% chance of winning $\$ 0$ ), or a $100 \%$ chance of winning $\$ 50$. Both bets have the same inherent value if taken a large number of times (\$50), however one's preference of one over the other may differ, depending on their appetite for risk. Identifying this helps to define how the performance (in this case money vs. probability) maps to utility.
} 
The MAU function $(U(x))$ can be derived using the multiplicative form, as

$$
U_{\text {mult }}(x)=\frac{1}{K}\left(\prod_{i=1}^{n}\left[K k_{i} U_{i}\left(x_{i}\right)+1\right]-1\right)
$$

where $k_{i}$ is the weight applied to the attribute $i$, and $K$ is a scaling constant that can be found through solving

$$
1-K=\prod_{i=1}^{n}\left(1+K k_{i}\right)
$$

The simpler, additive form is defined by

$$
U_{a d d}(x)=\sum_{i=1}^{n} k_{i} U_{i}\left(x_{i}\right)
$$

Determination of the set $k_{i}$ can be difficult, but again it is typically established using lottery questions during interview [19]. It is important to understand the relationship between the number of attributes and their relative influence on the overall utility. 


\section{2. $\quad$ Spacecraft Model}

Models that capture the transition between operational states representing different levels of failure are an important mechanism for understanding through-life behaviours. Such models have been applied to successfully evaluate the multi-state failures associated with spacecraft at the system and sub-system level [21] and [22]. For a spacecraft with an inter-satellite networking capability, there exists, in addition to the nominal "collect" and "deliver" capabilities, another function. This is its ability to "relay" information, which not only provides a higher nominal utility via increased functionality, but introduces four degraded operational states that are not available to a non-networked system. This is described in Figure 3, where the assumption is made that additional hardware is required for ISN, thus offering additional resilience*.

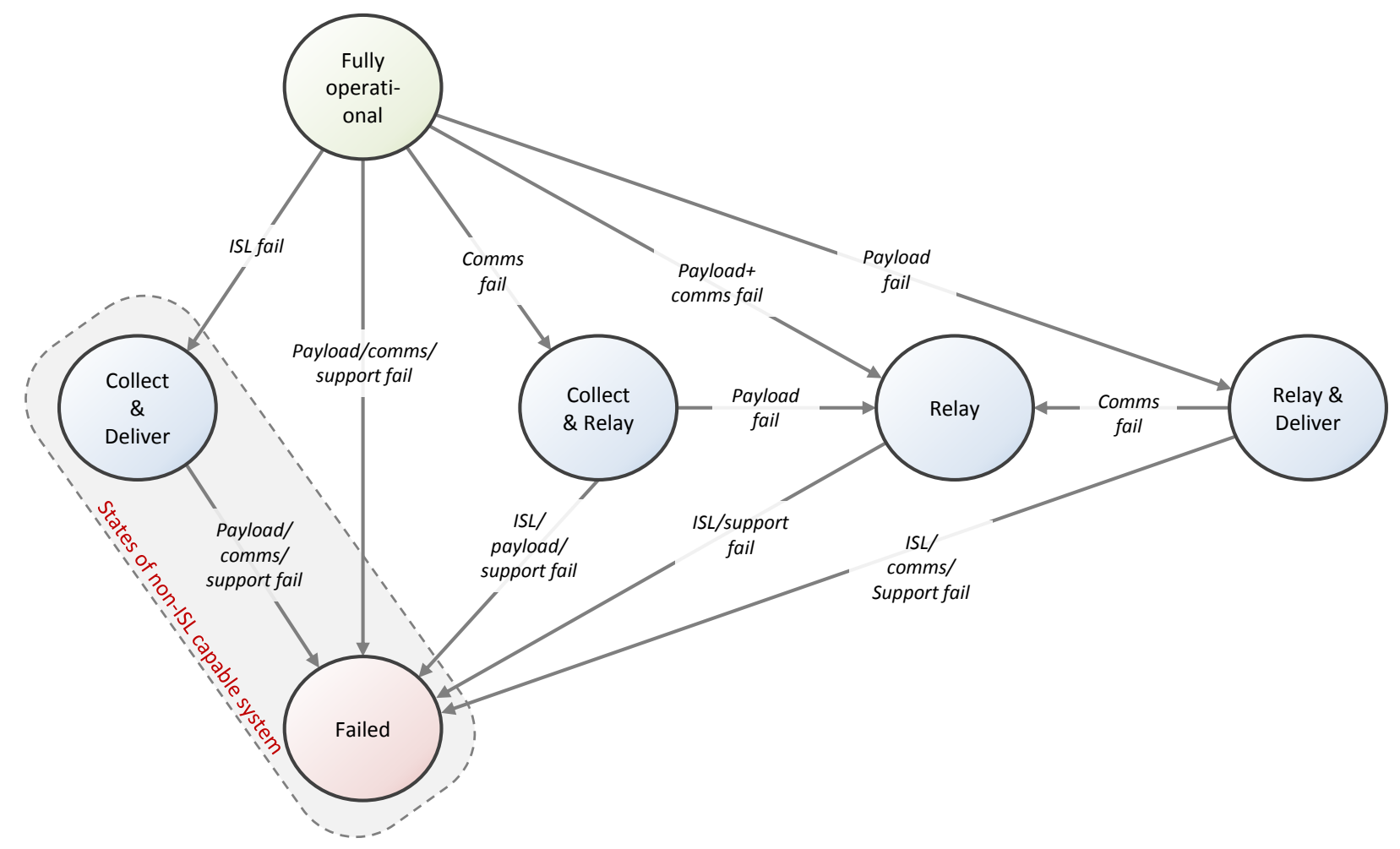

Figure 3 - State transition Markov chain diagram for a networked and non-networked system

Figure 3 shows the state possibilities being considered here, including the partially failed states and the potential transitions to and from each state. All partially- or fully-failed states could transition to a more operational state if a repair/replace service is available, however this is not addressed in this work due to the lack of available servicing to space assets. The Markov chain in Figure 3 can also be represented as a probability state-transition matrix of the form

\footnotetext{
${ }^{*}$ While it is technically feasible that an ISN capability is implemented using the same hardware as used between the satellite and ground station, for the purposes of this work an ISL is assumed to require additional hardware. This is considered a reasonable assumption given the likelihood of differences in link frequency and potential pointing demands.
} 


$$
P\left(t_{k}\right)=\left[\begin{array}{cccccc}
p_{11} & p_{12} & p_{13} & p_{14} & p_{15} & p_{16} \\
0 & p_{22} & 0 & 0 & 0 & p_{26} \\
0 & 0 & p_{33} & 0 & P_{35} & p_{36} \\
0 & 0 & 0 & p_{44} & p_{45} & p_{46} \\
0 & 0 & 0 & 0 & p_{55} & p_{56} \\
0 & 0 & 0 & 0 & 0 & 1
\end{array}\right]
$$

where $P\left(t_{k}\right)$ is the transition matrix at time $t_{k}$, and $p_{i j}$ is the probability that the system will transition from state $i$ to state $j$ during time period $t_{k+1}-t_{k}$. Note that the number of non-zero entries along the row direction indicates the number of states to which a state (defined by row number) can transition, and along the column direction the number of states from which a state (defined by column number) can be transitioned. Recall that the discrete-time form of Markov chains is employed, whereby the matrix $P$ may differ over the lifetime. The probability of being in each state at time $t_{k}$, given the likelihood of being in each initial state, as defined by the vector $\pi^{(0)}$, is

$$
\pi^{\left(t_{k}\right)}=\pi^{(0)} P(0) P(1) \ldots P\left(t_{k}-1\right)
$$

Consider a satellite with failure probabilities for the payload, ISN system, communication system and critical support systems, of 5\%,4\%, 3\% and 5\% respectively, in any given year. These probabilities have been chosen in order to illustrate the methods presented in this article and are not necessarily reflective of real failure probabilities. Furthermore, while the probabilities used in this example are constant over the system lifetime, it should be noted that this is not a limitation of the method, such that dynamic probabilities could be used without the need for modification. 
The state probability plot is shown for both a non-networked system, i.e. one without an ISN system (Figure 4), and a networked system (Figure 5).

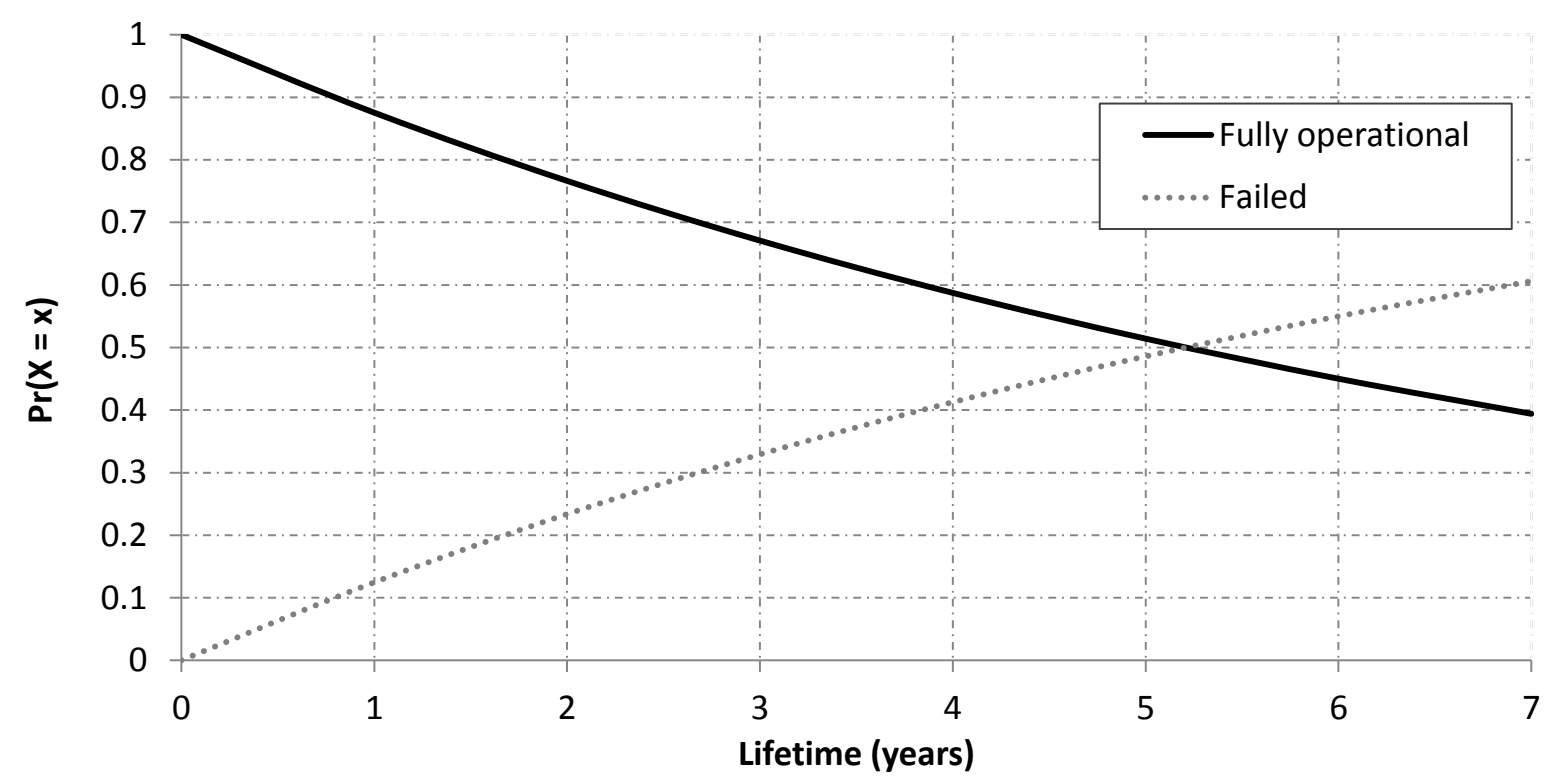

Figure 4 - State probability of a system without networking capability

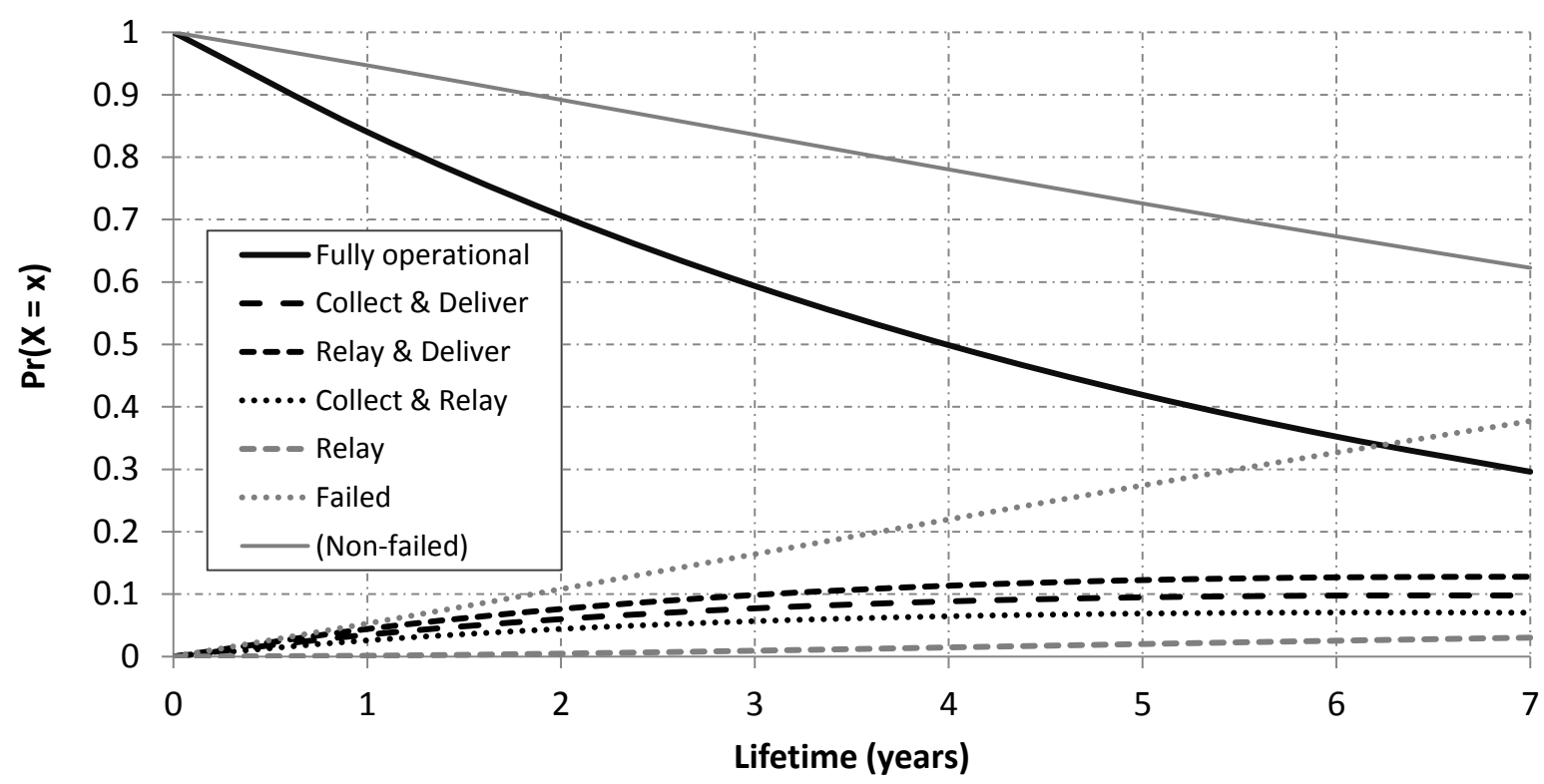

Figure 5 - State probability of a system with a networking capability

Figure 4 and Figure 5 show that, for this specific set of transition probabilities, a networkingcapable system is equally likely to remain in either a fully operational state or a collect \& deliver state, as a non-networked system is to remain in its fully operational state (which is, by definition, a collect \& deliver state). And, a networking-capable system is less likely to enter into a fully failed state (as illustrated by the "Non-failed" line), since there are intermediate, degraded states that are 
reachable. In this example, after 7 years, the networked system is $\sim 23 \%$ less likely to be in a fully failed state, than a non-networked system.

While the failure likelihood, shown in Figure 4 and Figure 5, is exaggerated above that seen in real data for illustrative effect, it is clear that there may be value in incorporating inter-satellite networking with regards to lifetime utility. This will come at a cost, both in financial terms and complexity.

\section{Network Model}

Determination of a system's expected state probability over its lifetime may be of interest from an academic perspective, but it provides limited insight into how a mission's utility will be affected by failure. This problem can be tackled either semi-analytically or numerically, the choice of which is dependent on factors including network size, state complexity and available computational resource for the analysis. While the discussion thus far has surrounded failure effects on a single node and its various states, when considering a network it is the state taken by each node combined to form a network-state that is of greater importance. A network-state can be described as a particular set of states in which each node that comprises the network resides. The fully operational network-state will constitute all nodes in the network being without defect, while other network-states may feature specific nodes with some level of failure present. 


\subsection{Network Types}

The composition of a network in terms of node properties can be considered a continuous spectrum, however three discrete families are defined in Table 1, which can be said to exist at the extremes of this scale.

Type

Definition

Graphic

A set of similar nodes that exhibit the same possible state
transitions, with the same probability of transition. The
influence on the network from each node, i.e. the effect of a
state transition on one particular node, is equal to that from
any other node.
Homogenous
similar orbits, evenly distributed around the Earth.
As for a homogeneous network, but with nodes exhibiting
different levels of influence over the network.
Example, a constellation of satellites of similar design, most
of which are in an equatorial orbit but with one, or a few
satellites in an elliptical equatorial orbit offering exclusive
communication with, and observation of, low-latitude
regions.
Anomogenous network of dissimilar nodes, exhibiting different state
transition possibilities and having different levels of
influence over the network.
Example, a network comprising a set of Earth observation
satellites, the International Space Station (ISS), and the
European Data Relay System (EDRS)

Table 1 - Network types for the analysis of network state transitions

In reality, it is likely that a network would be described as some combination of these network types, but the introduction of these network types enables characterisation of the network-state modelling approach.

\subsection{Permutations versus Combinations}

In order to evaluate how network states evolve over their lifetime, we must understand the different states in which the network can reside at any particular time. In the case of nonhomogeneous networks, the set of network states can be described using permutations (Section 
3.2.1), while for homogeneous networks, the network states can be described by combinations (Section 3.2.2). Heterogeneous networks require evaluation of each set of individual node states in order to define the full set of network states, which cannot be simplified further.

\subsubsection{Permutations}

Consider a network of $N$ nodes, where $\mathcal{N}=\left\{n_{1}, n_{2}, \ldots, n_{N}\right\}$ is the set of nodes, to which the set of states $\mathcal{M}=\left\{m_{1}, m_{2}, \ldots, m_{M}\right\}$ are attributed, such that a node can reside in any one of $M$ states. A network-state $S_{i}$ is defined as a unique set of node-state pairs for all nodes, where $S_{i}=$ $\left\{s_{1}, s_{2}, \ldots, s_{N}\right\}$, and $s_{j} \in \mathcal{M}$ is the state of node $j$. This can be defined formally as an $N$-permutation of the multi-set $\mathcal{M}$ [23], where the number of permutations, and thus the number of network-states, is equal to $M^{N}$. A simple two-node, $\mathcal{N}=\{1,2\}$, three state, $\mathcal{M}=\{a, b, c\}$, network can be used to illustrate this concept in Table 2.

\begin{tabular}{llllllllll}
\hline \hline Network State \# & 1 & 2 & 3 & 4 & 5 & 6 & 7 & 8 & 9 \\
\hline Node 1 state & $a$ & $b$ & $c$ & $a$ & $b$ & $c$ & $a$ & $b$ & $c$ \\
Node 2 state & $a$ & $a$ & $a$ & $b$ & $b$ & $b$ & $c$ & $c$ & $c$ \\
\hline
\end{tabular}

Table 2 - Network states for a 2-node, 3-state network with permutations

While it is useful to understand the utility achieved by the network in each network-state, for semianalytical derivation of the expected utility over the lifetime, this can quickly become impractical as the number of nodes and states increase. For example, the number of unique network-states for 10 nodes, each with 6 state possibilities, is over 60 million. To overcome this limitation, it may be possible to exploit combinations, instead of permutations.

\subsubsection{Combinations}

For networks that exhibit some degree of uniformity amongst the nodes, in terms of their influence on network performance*, a fundamental simplification can often be made. This is to represent the network-states as a non-repeated, un-ordered set of node-states. For the same example as above, with a 2-node $\mathcal{N}=\{1,2\}$, three state, $\mathcal{M}=\{a, b, c\}$ system, the network states are (Table 3 ):

\begin{tabular}{lllllll}
\hline \hline Network State \# & 1 & 2 & 3 & 4 & 5 & 6 \\
\hline Node 1 (or 2) state & $a$ & $b$ & $c$ & $b$ & $c$ & $c$ \\
Node 2 (or 1) state & $a$ & $a$ & $a$ & $b$ & $b$ & $c$ \\
\hline \hline
\end{tabular}

Table 3 - Network states for a 2-node, 3-state network with combinations

\footnotetext{
* For example, a homogenous Walker constellation [25] of identical satellites, all carrying out similar functions, could be considered a uniform network, while one comprising the international space station (ISS), a polar-orbiting Earth observation platform and a geostationary communication satellite, each with a very different function, could not.
} 
Using the same notation as in the previous section, the number of network-state combinations, for a network made up of $N$ nodes, each with $M$ node-states, can be found through application of the combinatorial problem, multiset counting [23]. Formally, an $N$-combination of the multiset $\mathcal{M}$, as defined above, is

$$
\left(\begin{array}{c}
N+M-1 \\
N
\end{array}\right)=\frac{(N+M-1) !}{N !(M-1) !}
$$

where the left-hand side of equation 3.1 uses standard binomial coefficient notation. In other words, this can be defined as the number of $N$-combinations of $M$ distinct objects, each with unlimited supply. To illustrate the problem with analysing large networks defined by permutations, the number of permutations (repeated) and combinations (non-repeated), for a 6-state network of various node-counts, is shown in Figure 6.

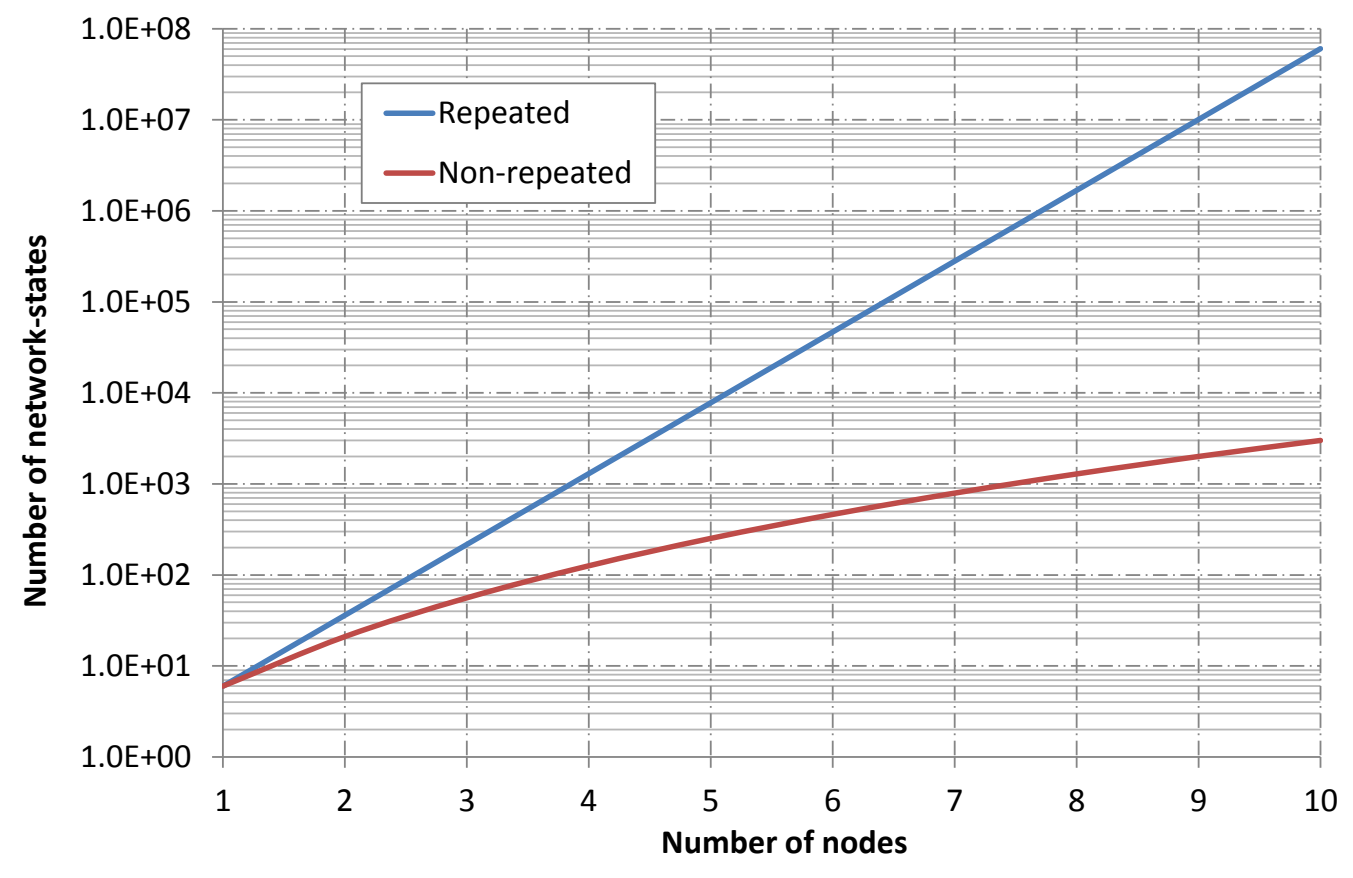

Figure 6 - Number of permutations (blue) and combinations (red) of network-states for a six state network

It is clear that for large networks, exploiting non-repeated network-states is not only beneficial, but necessary in the case of desktop computation facilities, for semi-analytical assessment of life-cycle utility. It must, however, be reiterated that this is a simplification, and should be used with caution on networks that are not strictly homogeneous in the sense of node-influence. In the event of a highly heterogeneous network, a numerical approach is recommended, whereby Monte-Carlo simulations are executed in order to derive an estimate for the expected utility at different times over the mission lifecycle. 


\section{Mission Case Study}

A multi-platform mission is evaluated where mission performance degrades over time. Comparison of mission performance is made between a traditional, non-networked version of the system, and one with ISN capability to illustrate the potential lifetime value of ISN. A semi-analytical assessment is performed (Section 4.3), using Markov chains, to estimate lifetime utility of the systems, subject to failures. These results are then further corroboration using Monte Carlo simulation assessment (Section 4.4).

\subsection{Concept of Operations}

Consider a constellation of seven small satellites of similar design (such that combinations can be used to describe the network states) that collect data stochastically over their orbit and download it to a number of ground stations distributed around the Earth. This type of mission could be considered representative of, say, an Earth observation (EO) system, or a machine to machine (M2M) messaging service.

The reliability, defined as the probability of being operational, of each sub-system over a seven-year lifetime is shown in Figure 7. The feature of beginning of life failure on the communications, ISL and other sub-systems is clear from this data, however payloads tend to exhibit a more consistent decay in reliability over the lifetime.

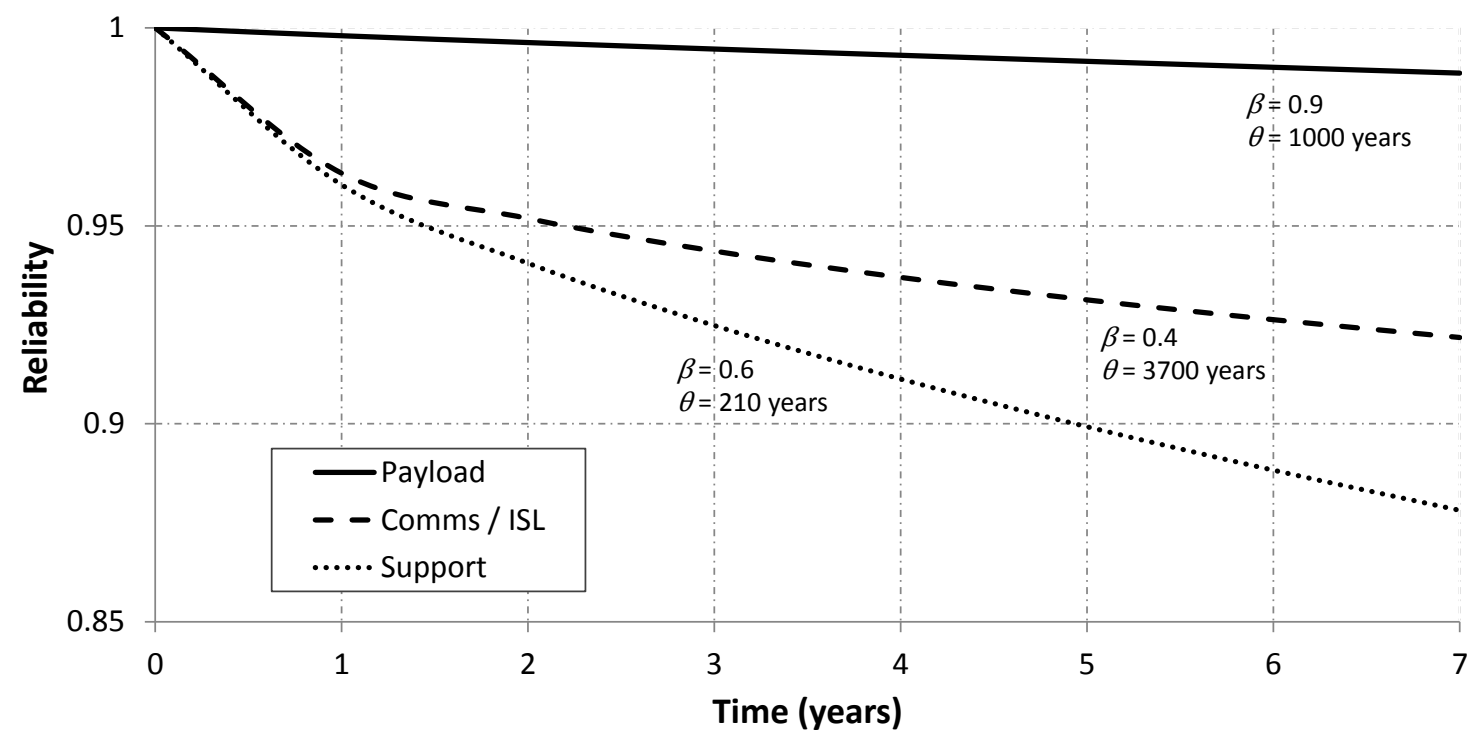

Figure 7 - Reliability (probability of being functional) of sub-systems

The hypothetical satellite network being analysed here is one that is assumed to exploit opportunistic launch, something that has become increasingly popular over the past decade due to the relatively high launch cost savings compared with dedicated orbit placement. This approach is of course not appropriate for certain systems that demand specific orbital parameters, but it is 
assumed that for this mission, relative orbital position between each asset is not important. It is considered possible to transfer data between satellites when their respective distance is below some threshold and data routing is carried out using the Spae routing algorithm, described in [24]. This algorithm ensures low data latency through delay-tolerant networks (DTNs) with deterministic mobility patterns, such as that exhibited in this case study. The simulation parameters are summarised in Table 4.

\begin{tabular}{|c|c|c|c|}
\hline Variable & Quantity & Units & Comment \\
\hline Orbit altitude & {$[400,800]$} & $\mathrm{km}$ & $\begin{array}{l}\text { Random for each satellite in each state-specific } \\
\text { simulation (uniform distribution) }\end{array}$ \\
\hline Orbit inclination & {$[20,100]$} & deg. & $\begin{array}{l}\text { Random for each satellite in each state-specific } \\
\text { simulation (uniform distribution) }\end{array}$ \\
\hline $\begin{array}{l}\text { Payload Data } \\
\text { lifetime }\end{array}$ & 120 & $\min$ & $\begin{array}{l}\text { Time, from acquisition, until data is discarded if not } \\
\text { yet downloaded to ground }\end{array}$ \\
\hline ISL range & 5000 & $\mathrm{~km}$ & $\begin{array}{l}\text { Threshold distance between satellites, above which } \\
\text { ISN cannot occur }\end{array}$ \\
\hline ISL data rate & 50 & $\mathrm{Mb} / \mathrm{s}$ & Indicative of S-band communication \\
\hline $\begin{array}{l}\text { Space-ground } \\
\text { data rate }\end{array}$ & 50 & $\mathrm{Mb} / \mathrm{s}$ & Indicative of S-band communication \\
\hline $\begin{array}{l}\text { Simulation } \\
\text { duration }\end{array}$ & 1 & day & Operational scenario duration \\
\hline $\begin{array}{l}\text { Simulation time } \\
\text { step }\end{array}$ & 10 & $\mathrm{~s}$ & Numerical simulation parameter \\
\hline $\begin{array}{l}\text { No. simulations } \\
\text { per state }\end{array}$ & 30 & \# & For each network state possible \\
\hline
\end{tabular}

Table 4 - Mission parameters and bounds

The constellation is considered to have access to a ground station network comprising assets in Kourou (Lat $5.3^{\circ} \mathrm{N}$, Lon $52.8^{\circ} \mathrm{W}$ ), Perth (Lat $31.8^{\circ} \mathrm{S}$, Lon $115.9^{\circ} \mathrm{E}$ ), Svalbard (Lat $78.2^{\circ} \mathrm{N}$, Lon $15.4^{\circ} \mathrm{E}$ ), South Point (Lat $19.0^{\circ} \mathrm{N}$, Lon $155.6^{\circ} \mathrm{W}$ ) and Hartebeesthoek (Lat $25.9^{\circ} \mathrm{S}$, Lon $27.7^{\circ} \mathrm{E}$ ). Visibility of the ground stations to each satellite is dependent on the satellite orbital inclination, but will be assumed visible if the satellite is more than $10^{\circ}$ above the horizon.

\subsection{Mission Utility}

Mission success is a combination of the following attributes:

- The amount of data downloaded (data volume).

- The amount of data lost (delivery ratio: ratio of collected to delivered data).

- The latency (time from acquisition to delivery) associated with the delivered data. 
A multi-attribute utility (MAU) function is used to measure the performance of the systems and directly compare their respective values. The attributes considered important in this hypothetical example are summarised in Table 5, along with their relative contribution to the MAU function, for which a linear form is used.

\begin{tabular}{lccc}
\hline \multicolumn{1}{c}{ Attribute } & Objective & Limits & Weighting \\
\hline Data latency (mean) & minimum & {$[20,100]$ mins } & 2 \\
Data latency (variance) & minimum & {$[20,40]$ mins } & 1 \\
Delivery ratio (mean) & maximum & {$[0,1]$} & 1 \\
Data volume (mean) & maximum & {$[0,7] \mathrm{Gb} /$ day } & 2 \\
\hline \hline
\end{tabular}

Table 5 - Attributes considered in the multi-attribute utility calculation

Noteworthy is the inclusion of a data volume attribute, which is an important consideration when failures are involved. Consider the independent (non-networked) system in question: None of the other attributes (i.e. data latency or delivery ratio) would be affected by the failure of a payload or communications system, since in both instances the failed node is considered removed from the network. As a result, data volume must be considered as a method of quantifying the effects of failure in that case.

\subsection{Semi-Analytical Assessment}

The added lifetime utility of inter-satellite networking can be found in a semi-analytical fashion by comparing, to an equivalent non-networked system, the utility associated with each network-state over the lifetime, scaled by the likelihood of that state existing. This expected utility $\left(U_{k}\right)$, at time $k$, is

$$
U_{k}=\sum_{j=1}^{m} P_{k j} U_{j},
$$

where $P_{k j}$ is the probability of being in state $j$ at time $k$ and $U_{j}$ is the utility of the system in state $j$. Given a set of states in which the network can reside, it is necessary to identify the state-specific utilities a priori, using either permutations or combinations, as appropriate. For large state-spaces, this is perhaps not possible, such that a sample might instead be analysed, and the utility for those neglected from the initial analysis, estimated via heuristic-interpolation. In this case study, utility in all states is evaluated.

Separately from the calculation of network-state utility, it is necessary to derive the probability of being in each network state over the lifetime of the mission. This is achieved using Markov chains, as described in Section 1.1. A time-discrete approach is taken to enable assessment of non- 
homogeneous networks, where resilience varies over time based on the Weibull parameters in Figure 7.

The utility for both the networking-capable and independent systems, for each of their respective network states, is derived through numerical simulation. At discrete 1-year intervals over the lifetime, the expected utility is calculated using equation 4.1, shown in Figure 8.

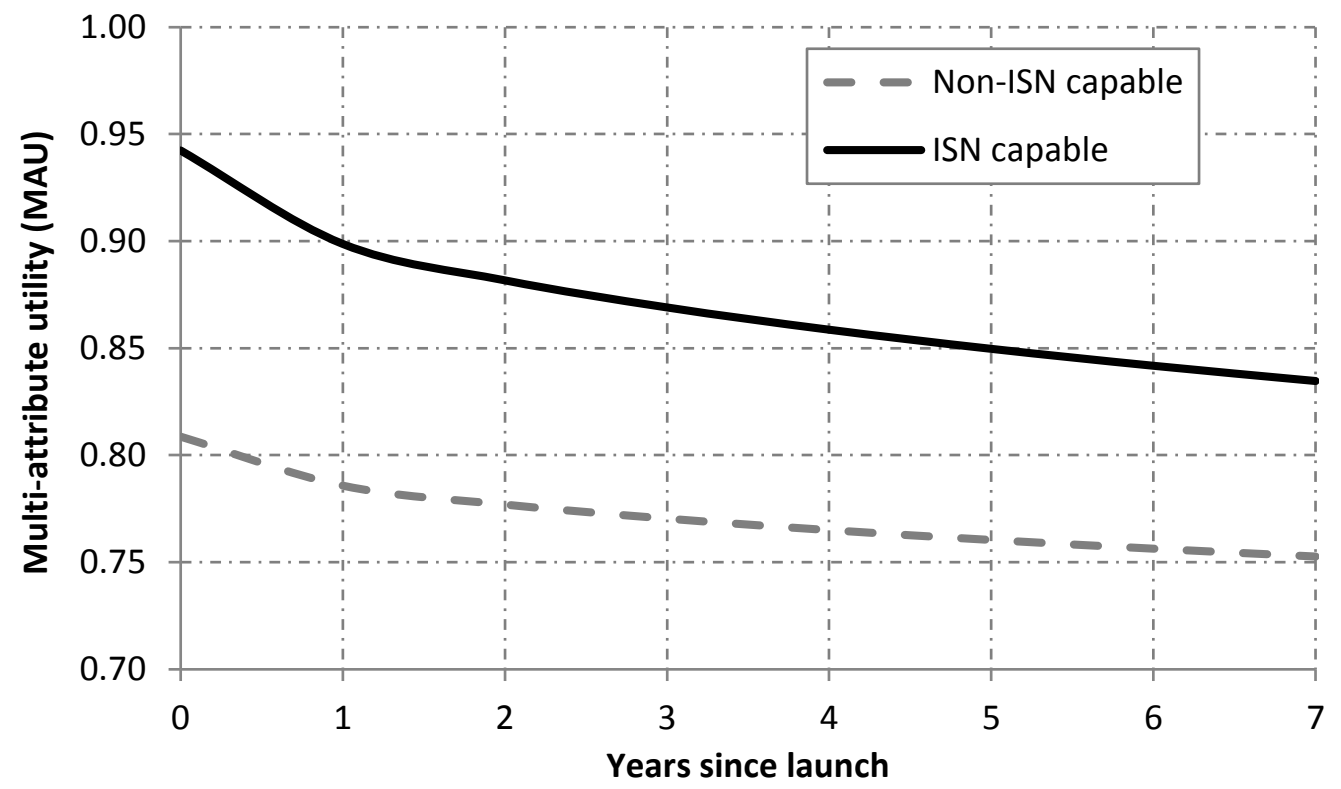

Figure 8 -Multi-attribute utility (MAU) of the independent and networked systems over the lifetime

Perhaps surprisingly, the reduction in utility over the lifetime is greater in the case of the networking-capable system (from 0.94 at the beginning of life, to 0.83 at end of life), than for the non-networked system (from 0.81 at beginning of life to 0.75 at end of life), despite the increased likelihood of each node remaining in a non-failed state due to the additional relay functionality. According to the reliabilities of the payload, communications system and ISN system (Figure 7), it is clear that the likelihood of payload failure is significantly lower than for the communications and ISN systems. This means that in the networked case, it is more likely that nodes will be relied upon to download other's data (whose communication system has failed), than for a reduced amount of data to be collected. Consequently, the networked system suffers a reduction in delivery ratio and an increase in data latency as failures occur. In the independent system, however, neither of these attributes are affected by a communications system or payload failure, since in both cases the offending node is simply removed from the network completely (fully failed).

What is clear, however, is that despite the greater reduction in delivered data, the volume remains significantly higher throughout in the ISN case and would continue to do so irrespective of lifetime, as shown in Figure 9. 


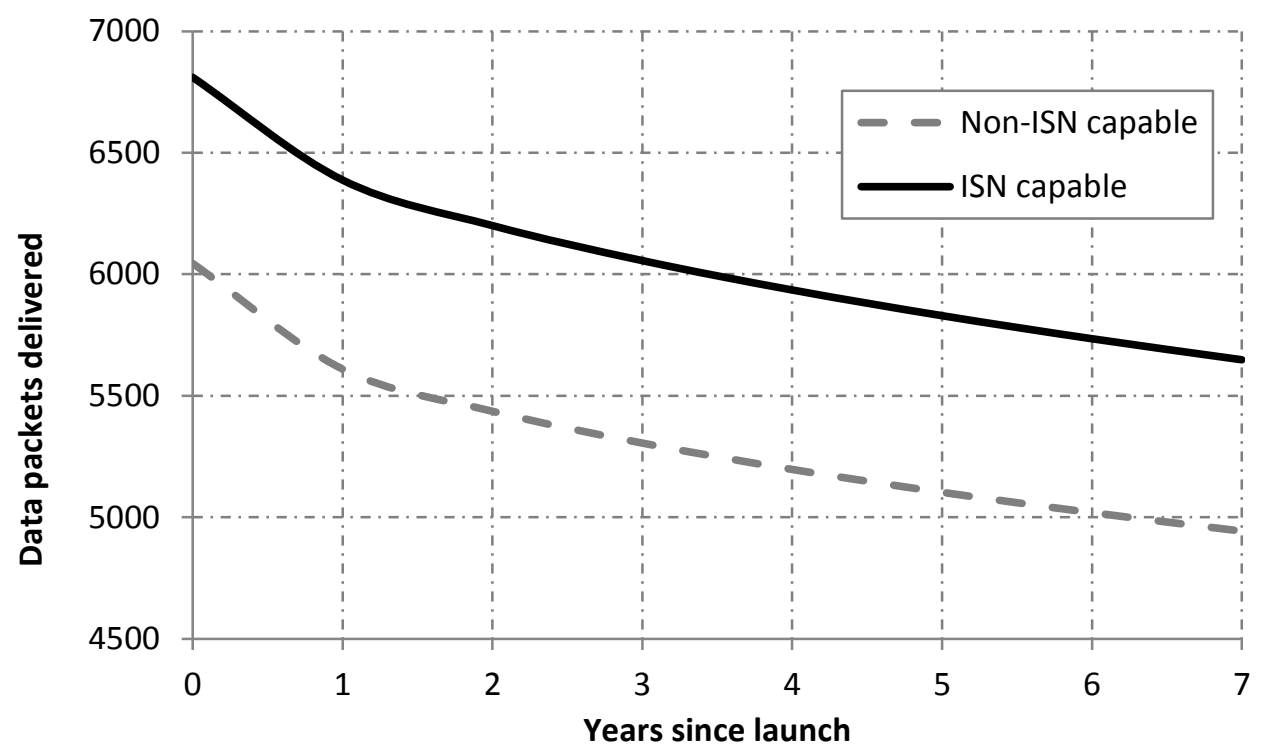

Figure 9 - Expected volume of delivered data (per day) over mission lifetime

The fully operational utility and expected lifetime utility (average over the entire seven year lifetime) are shown in Table 6 where it is seen that while ISN offers an in increased probability of remaining in a fully- or partially-operational state, it does not necessarily result in a reduced magnitude loss in utility over the lifetime. As such, although in a networking-capable system each platform exhibits a more graceful degradation in function, and the overall utility remains greater than a non-networked system, unexpectedly the system itself exhibits a less graceful degradation than a non-networked system.

\begin{tabular}{lccc}
\hline \hline \multicolumn{1}{c}{ System } & Nominal utility & Lifetime utility & $\begin{array}{c}\text { Reduction in } \\
\text { utility }\end{array}$ \\
\hline Independent & 0.809 & 0.772 & $4.6 \%$ \\
Networked & 0.943 & 0.872 & $7.5 \%$ \\
\hline \hline
\end{tabular}

Table 6 - Nominal and lifetime utility

Consider now the other, single attribute utilities. An increase in data latency (Figure 10) and a reduction in delivery ratio (Figure 11) is experienced, which is expected given the findings above. Those platforms with functional communication systems remaining, will suffer with requests from other platforms wanting use of their capabilities for delivery of their data. Again, in an independent system, any failure removes the platform from the network, such that they are not then asked to download data from other sources. 


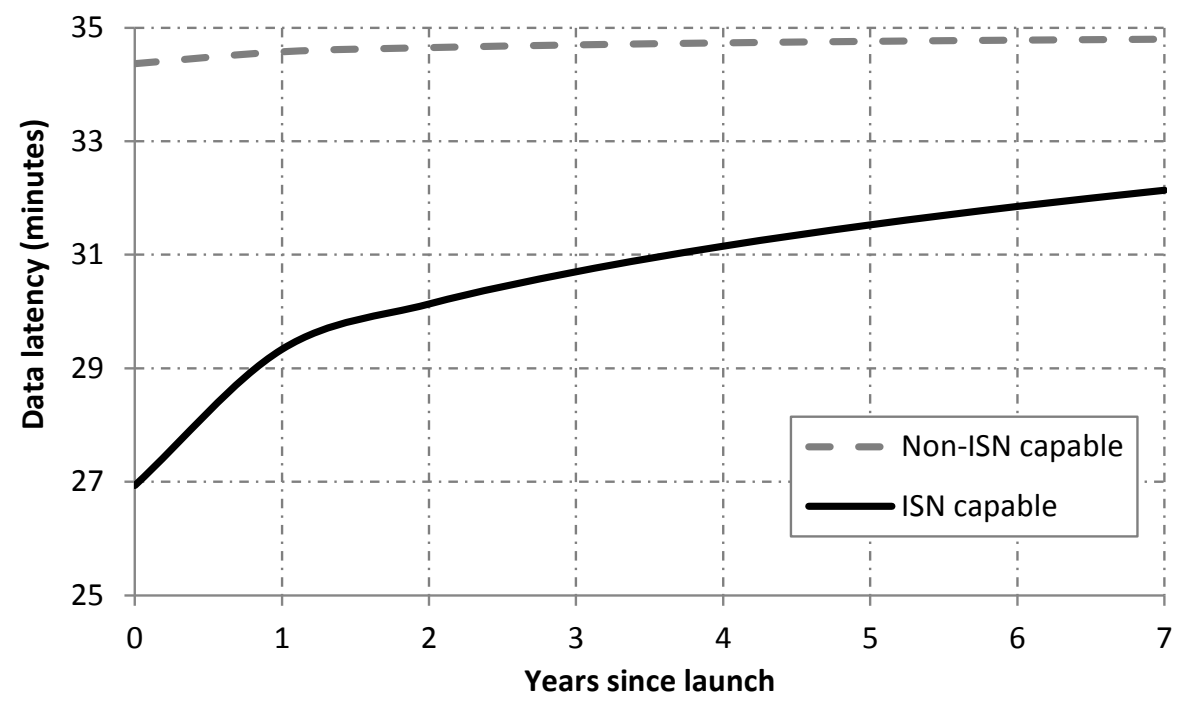

Figure 10 - Average (mean) data latency vs. lifetime

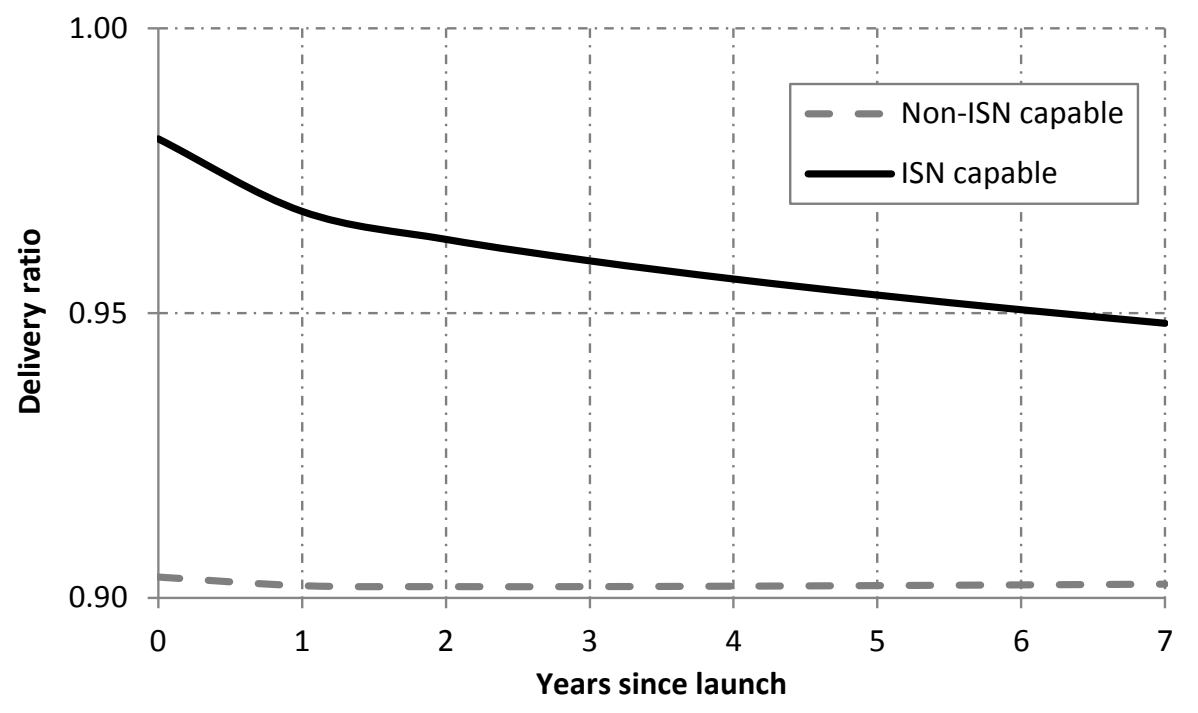

Figure 11 - Average (mean) data delivery ratio vs. lifetime

As a result of the findings from these simulations, it is clear that should failures occur that affect the balance between data collection and data delivery, the operational parameters should be re-defined so as not to overwhelm platforms with remaining download capability.

\subsection{Numerical Corroboration}

In order to corroborate the above result, a Monte Carlo simulation is carried out in which failure of the payload, communication system, other sub-systems and, in the case of the ISN-capable platforms, ISN system are induced at discrete times through a seven-year lifetime. Failure occurs according to the same probabilities as used in the semi-analytical approach, based on the Weibull 
parameters in Figure 7. One-hundred thousand simulations are carried out for both the networking-capable, and for the independent systems.

The general process that is executed during the Monte Carlo analysis is illustrated by the algorithm in Table 7, where $Y$ is the number of MC simulations, $T$ is the number of time intervals (each equal to 1 year), $N$ is the number of nodes in the network, $\mathcal{S}=\left\{s_{1}, s_{2}, \ldots, s_{|\mathcal{S}-1|}, s_{|\mathcal{S}|}\right\}$ is the set of possible network states, $\mathcal{M}=\left\{m_{1}, m_{2}, \ldots, m_{M-1}, m_{M}\right\}$ is the set of node states, $P=\mathbb{R}^{M \times M \times T}$ is the node state transition matrix with entry $P_{i j k}$ being the probability of a node in state $i$ transitioning to state $j$ at time $k, S_{i j}$ is the state in which the network resides at time $i$ and simulation $j$, with the initial state being fully operational in both the independent and networking-capable system examples and $m_{j k}$ is the state in which node $j$ resides in at time $k$, which is dictated by $S$.

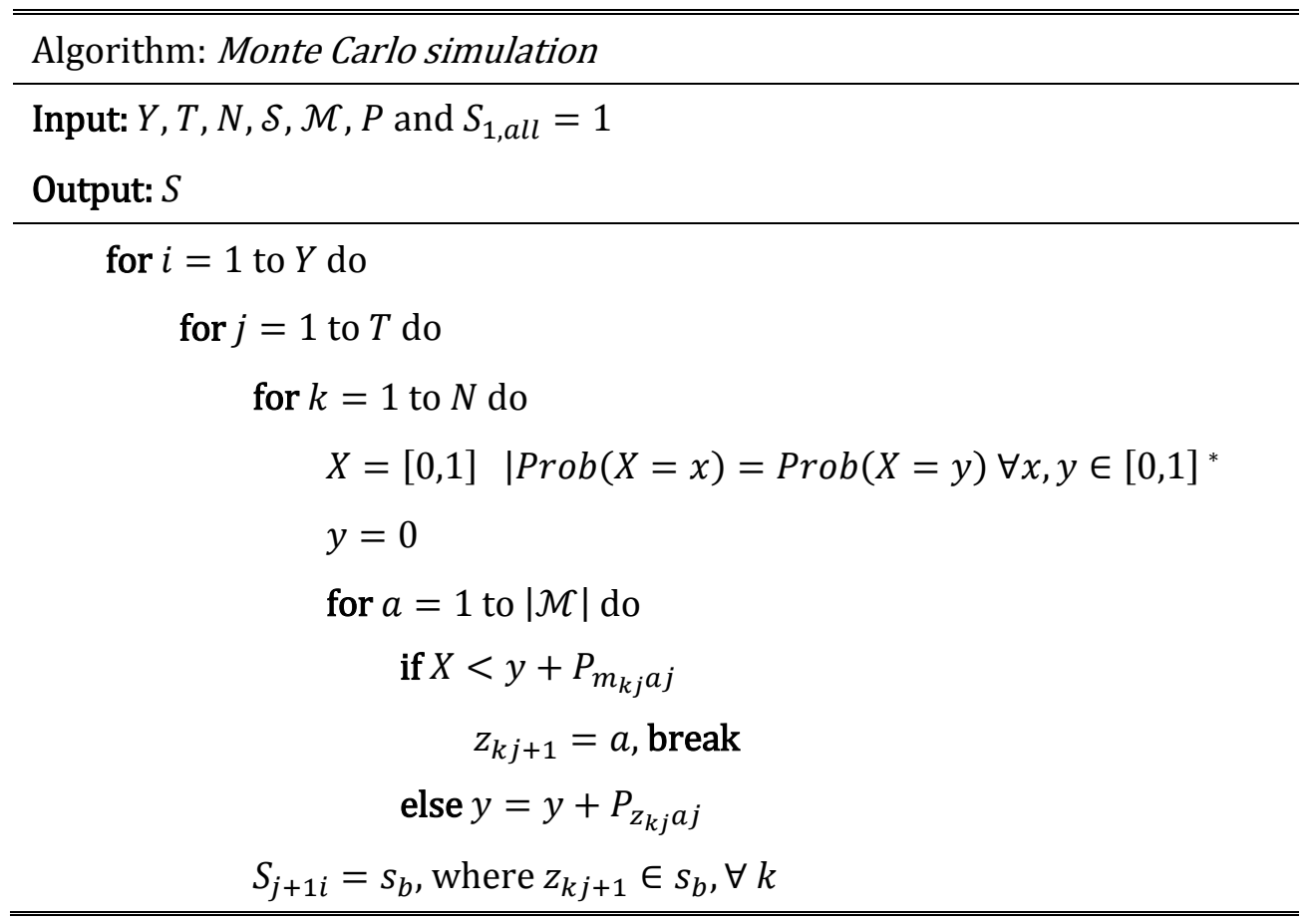

Table 7 - Algorithm for Monte Carlo simulation

The output from this analysis, the array $N$, defines the state of the network at the start of each time interval, for each of the Monte Carlo simulations. The multi-attribute utilities associated with these states can then be applied to identify the utility over the lifetime, for each simulation.

Results of utility over the seven-year lifetime from the Monte Carlo analysis are shown in Figure 12. A similar change in utility to that found in the previous section is seen, which confirms the accuracy of the Markov Chain approach introduced in this work. From the numerical approach, knowledge of the variance in results can also be identified, with error-bars showing the $95^{\text {th }}$ percentile. Note that the variance of the ISN-capable system drops to below that of the independent system, suggesting

\footnotetext{
${ }^{*}$ In other words, $X$ is a uniformly distributed continuous random variable between 0 and 1
} 
that there is potential for a real-terms lower utility to be realised, should no measures be put in place to modify the operations sufficiently.

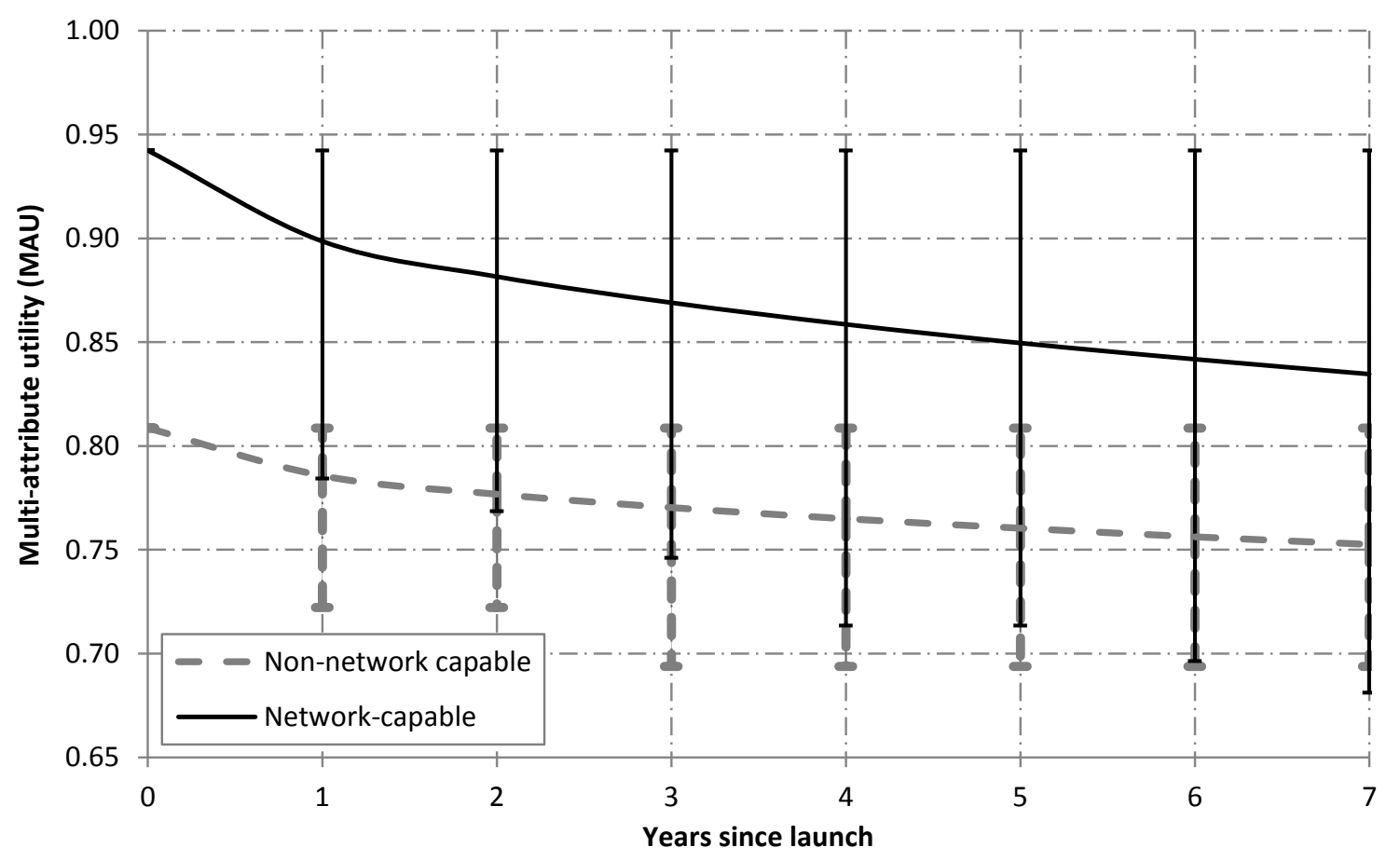

Figure 12 - Monte Carlo corroboration results

\section{Conclusions}

By modelling a spacecraft as either a networking-capable (i.e. one with an inter-satellite networking capability) or a non-networking-capable system, and capturing state transitions via a Markov model, it is shown that the presence of inter-satellite links renders a platform less likely to enter a fully failed state during its lifetime. Simulation of a seven-satellite, seven-year mission shows that despite this added resilience, should anomalies occur that affect the balance between data collection and data delivery, overall value may decrease by a greater proportion on a network with ISN. This can be attributed to the increased reliance on platforms with functional downlink systems being used for delivery of other platform's data, for whom the on-board downlink capability has been lost. This makes clear the need for continual assessment of the mission operations, such that specific nodes do not become overwhelmed in the event of degradation of other nodes in the network. Modifications to operational parameters should, in these cases, be considered. 


\section{References}

[1] J. a. Fraire and P. a. Ferreyra, "Assessing DTN architecture reliability for distributed satellite constellations: Preliminary results from a case study," in 2014 IEEE Biennial Congress of Argentina (ARGENCON), 2014, pp. 564-569, doi: 10.1109/ARGENCON.2014.6868551.

[2] H. Cruz-Sánchez, L. Franck, and B. Andre-Luc, "Use of Store and Forward Metrics for ServiceOriented Routing in Satellite Constellations," in Satellite and Space Communications, IWSSC, 2008, pp. 80-84, doi: 10.1109/IWSSC.2008.4656753.

[3] C. Caini and R. Firrincieli, "Application of Contact Graph Routing to LEO satellite DTN communications," in 2012 IEEE International Conference on Communications (ICC), 2012, pp. 3301-3305, doi: 10.1109/ICC.2012.6363686.

[4] R. Diana, E. Lochin, L. Franck, C. Baudoin, E. Dubois, and P. Gelard, "A DTN routing scheme for quasi-deterministic networks with application to LEO satellites topology," in IEEE Vehicular Technology Conference (VTC), 2012, doi: 10.1109/VTCFall.2012.6399087.

[5] C. D. Jilla, D. W. Miller, and R. J. Sedwick, "Application of Multidisciplinary Design Optimization Techniques to Distributed Satellite Systems," J. Spacecr. Rockets, vol. 37, no. 4, pp. 481-490, 2000, doi: 10.2514/2.3589.

[6] M. G. Richards, A. M. Ross, N. B. Shah, and D. E. Hastings, "Metrics for Evaluating Survivability in Dynamic Multi-Attribute Tradespace Exploration,” J. Spacecr. Rockets, vol. 46, no. 5, pp. 1049-1064, Sep. 2009, doi: 10.2514/1.41433.

[7] J.-F. Castet and J. H. Saleh, "Satellite and satellite subsystems reliability: Statistical data analysis and modeling," Reliab. Eng. Syst. Saf., vol. 94, no. 11, pp. 1718-1728, Nov. 2009, doi: 10.1016/j.ress.2009.05.004.

[8] J.-F. Castet and J. H. Saleh, "On the concept of survivability, with application to spacecraft and space-based networks," Reliab. Eng. Syst. Saf., vol. 99, pp. 123-138, Mar. 2012, doi: 10.1016/j.ress.2011.11.011.

[9] J. H. Saleh and K. Marais, "Highlights from the early (and pre-) history of reliability engineering," Reliab. Eng. Syst. Saf., vol. 91, no. 2, pp. 249-256, Feb. 2006, doi: 10.1016/j.ress.2005.01.003. 
[10] H. McManus, M. Richards, A. Ross, and D. Hastings, “A Framework for Incorporating 'ilities' in Tradespace Studies," in AIAA SPACE 2007 Conference \& Exposition, 2007, pp. 1-14, doi: 10.2514/6.2007-6100.

[11] J. H. Saleh, E. Lamassoure, and D. E. Hastings, "Space Systems Flexibility Provided by OnOrbit Servicing: Part 1," J. Spacecr. Rockets, vol. 39, no. 4, pp. 551-560, Jul. 2002, doi: $10.2514 / 2.3844$.

[12] E. Lamassoure, J. H. Saleh, and D. E. Hastings, "Space Systems Flexibility Provided by OnOrbit Servicing: Part 2," J. Spacecr. Rockets, vol. 39, no. 4, pp. 561-570, Jul. 2002, doi: $10.2514 / 2.3845$.

[13] W. Stewart, "Section II - Markov Chains," in Probability, Markov Chains, Queues, and Simulation: The Mathematical Basis of Performance Modeling, 1st ed., Princeton University Press, 2009, pp. 191-375.

[14] G. F. Dubos and J. H. Saleh, "Comparative cost and utility analysis of monolith and fractionated spacecraft using failure and replacement Markov models," Acta Astronaut,, vol. 68, no. 1-2, pp. 172-184, Jan. 2011, doi: 10.1016/j.actaastro.2010.07.011.

[15] J.-F. Castet and J. H. Saleh, "Satellite Reliability: Statistical Data Analysis and Modeling," J. Spacecr. Rockets, vol. 46, no. 5, pp. 1065-1076, Sep. 2009, doi: 10.2514/1.42243.

[16] A. Mehrparvar, “Cubesat design specification,” 2014.

[17] M. Langer and J. Boumeester, "Reliability of CubeSats - Statistical Data, Developers' Beliefs and the Way Forward," Proc. 30th Annu. AIAA/USU Conf. Small Satell., pp. 1-12, 2016.

[18] J.-F. Castet and J. H. Saleh, "Single versus mixture Weibull distributions for nonparametric satellite reliability," Reliab. Eng. Syst. Saf., vol. 95, no. 3, pp. 295-300, 2010, doi: 10.1016/j.ress.2009.10.001.

[19] R. Keeney and H. Raiffa, Decisions with multiple objectives: preferences and value tradeoffs, 1st ed. Cambridge University Press, 1993.

[20] A. Ross and D. Hastings, "Multi-attribute tradespace exploration as front end for effective space system design," J. Spacecr. ..., vol. 41, no. 1, 2004, doi: 10.2514/1.9204.

[21] J. Castet and J. H. Saleh, "Beyond reliability, multi-state failure analysis of satellite 
subsystems : A statistical approach," Reliab. Eng. Syst. Saf., vol. 95, no. 4, pp. 311-322, 2010, doi: 10.1016/j.ress.2009.11.001.

[22] S. Engelen, E. Gill, and C. Verhoeven, "On the reliability, availability, and throughput of satellite swarms," IEEE Trans. Aerosp. Electron. Syst., vol. 50, no. 2, pp. 1027-1037, 2014, doi: 10.1109/taes.2014.120711.

[23] R. Brualdi, "Permutations and Combinations," in Introductory Combinatorics, 5th ed., Pearson Education, 2009, pp. 46-55.

[24] C. J. Lowe and M. MacDonald, "Resource-considerate data routing through satellite networks," J. Aerosp. Inf. Syst., vol. 14, no. 8, pp. 472-482, 2017, doi: 10.2514/1.i010423.

[25] J. Walker, "Continuous whole-earth coverage by circular-orbit satellite patterns," 1977.

\section{Funding}

This research was funded in part by the Engineering and Physical Sciences Research Council (EPSRC), through an Industrial CASE (iCASE) studentship (EP/1501681/1), and in part by the UK Space Agency through the International Partnership Programme Firesat project. 